\title{
Practice-based research in the United States
}

\author{
John W Beasley Wisconsin Research Network, Professor of Family Medicine, University of Wisconsin Department \\ of Family Medicine, Madison, Wisconsin, USA
}

\begin{abstract}
In the United States (US), Practice-Based Research Networks (PBRNs) are in rapid evolution. As in the United Kingdom (UK), within the last decade there has been widespread recognition that primary care is the 'undiscovered country' of health care, and that research in primary care is important and worthy of support.
\end{abstract}

Key words: family practice; general practice; primary care; research funding

\section{Environment}

The chaotic health care system in the US has had consequences for PBRN development. In the US, the funding of health care is a mixture of public and private funding to a great variety of practice structures. Primary care is provided by a wide array of clinicians including family physicians, internists, pediatricians, nurse practitioners and physicians' assistants. In addition, many medical subspecialists also provide some primary care services. At the same time, the division between hospital and office care is bridged by the fact that most primary care physicians care for patients in both settings. Only within the last decade has the US realized that primary care is important and also a field in need of a research base.

\section{History}

The transition of PBRNs from small 'research clubs' to larger and more sophisticated enterprises linked with each other began when the Ambulatory Sentinel Practice Network (ASPN), under the leadership of Paul Nutting, MD MSPH, became a catalytic force within the US, impacting on the policies of professional organizations and the government. This change in viewpoint was given further stimulus by the report of the Institute of Medicine which

Address for correspondence: John W Beasley, MD, University of Wisconsin Department of Family Medicine, 777 South Mills Street, Madison, Wisconsin 53715, USA.

(C)Arnold 2000 noted the need for PBRNs that would 'link...practitioners with those who carry out scientific investigations'. They further noted the need for dedicated support for the infrastructure of these networks (Donaldson et al., 1996). Another paper from the US Government's Agency for Health Care Policy and Research noted the "need for... infrastructures that link practitioners and researchers' (Lanier and Clancy, 1997).

During this same period, the American Academy of Family Physicians (AAFP) began a $\$ 7.7$ million research initiative which included money for fellowships, centers of excellence and PBRNs. This year, for the first time, the US Government's Agency for Healthcare Research and Quality (AHRQ) has offered funding for the development of network infrastructure. Fourteen competitive grants of $\$ 75000$ each planning purposes will be given, with special emphasis on the development of electronic methods of data collection and processing. Simultaneously, many academic departments of family medicine and other primary care specialties are seeking funds for PBRN development under federal programmes designed to stimulate the academic development of primary care. These departments make the argument that 'we need PBRNs as a research laboratory for our academic research mission'.

\section{Funding}

Historically, the greatest source of support for PBRNs is the hidden one of the time and energy of clinicians in communities who made networks 1463-4236(2000)PC019XX 
possible. Their contribution has been supported by professional groups such as the American Academy of Pediatrics and the AAFP which have supported research networks, the Pediatric Research in Office Settings (PROs) network in the case of the former and ASPN in the case of the latter. (More recently, with the closure of ASPN, the AAFP has undertaken to develop a new National Network for Family Practice and Primary Care Research NNFPPCR).

\section{Structures}

The diversity within the US health care system is reflected in US PBRNs which range from small, tightly knit groups (such as UprNet in Northern Michigan) to large loosely knit regional networks such as WReN in Wisconsin, with others being based on electronic medical record technologies (Practice Partner Research Group). Some large managed care groups have internal structures which, while responding to corporate needs, become de facto PBRNs. While the diversity of networks has caused some consternation among those who would like to know 'exactly what is a PBRN?', each model has its own particular strength and weakness. For example, one of the weaknesses of more tightly organized networks is the selection bias of participating physicians which becomes important if the physician or the practice is under study (Nutting et al., 1999).

\section{Goals}

It is no surprise that, along with the diversity of structures, there is also a great diversity of goals. Some networks are more 'pure', focusing specifically on ideas brought forth by community practitioners, and doing studies only when the whole network is likely to participate. Others have focused on studies for pharmaceutical companies. Still others, such as the WReN, have a broader set of goals which includes providing support for both academically based investigators and individual community clinicians (Beasley et al., 1991).

\section{Methods}

Network methods have shown similar diversity and creativity. Networks have used practically every known research method from double-blinded intervention trials, through the analysis of the clinical databases of managed care organizations, to data derived from electronic medical records and qualitative methods. ASPN and others initiated 'card studies' where specified bits of data are collected using paper and pencil methods - literally on cards which fit into a shirt pocket. In recent months, in part stimulated by the AHRQ grant offering, there has been considerable discussion of networks using hand-held computers (e.g. Palm, Pocket PCs) for data entry with these devices being linked by the Internet to study centres. This has the great potential of linking practices across geographic, specialty and organizational lines. These devices have the advantage of being useful for other purposes (such as drug information and phone numbers) as well as research, and thus clinicians will be likely to have them available while seeing patients.

\section{Linking networks}

For over a decade, it has been realized that there was a need to link PBRNs so that they could learn from each other and collaborate in studies where this would be useful. Thus in 1997 the Federation of Practice-Based Research Networks (FPBRN) was formed. It has three goals: (1) to advocate for practice-based research; (2) to build capacity for practice-based research; and (3) to foster collaboration and communication between networks.

Originally supported by ASPN, the FPBRN has recently found a home with the AAFP as part of their network support activities. While focusing for political purposes on family practice networks in the US, the remaining two goals led the FPBRN to seek out communication with other networks, both in other disciplines and in other countries. At this time there are 26 PBRN members of the FPBRN. Most of these are family practice-oriented and in the US, but networks in Canada, the UK, the Netherlands and Korea are included. Communications are by a newsletter and a listserver. The FPBRN presents annual workshops at the meeting of the North American Primary Care 
Research Group (NAPCRG), and is developing an inventory of PBRNs in the US and other countries.

\section{Accomplishments}

There is increasingly important literature coming from PBRNs (Nutting et al., 1996). Beyond that, however, there is another aspect to the accomplishments of PBRNs - that of a change in the culture of community practice so that the scholarly work of research will become part of primary care.

The combination of the recognition of the importance of primary care research, the increasing interest on the part of community clinicians and the availability of some federal funding for infrastructure may at last allow us to complete our health care research enterprise by adding the missing link of research at the level of the clinician, the clinical encounter and the community.

\section{Note}

The FPBRN may be contacted via Tom Stewart, American Academy of Family Physicians, 11400
Tomahawk Creek Parkway, Leawood, KS 66211, USA. Tel: 001-800-274-2237 or 001-913-9066000. Email: tstewart@aafp.org

\section{References}

Beasley, J.W., Smith Cox, N., Livingston, B.T. et al. 1991: Development and operation of the Wisconsin Research Network. Wisconsin Medical Journal 90, 531-37.

Donaldson, M.S., Yordy, K.D., Lohr, K.N. and Vanselow, N.A., Editors. 1996: Primary care: America's health in a new era. Washington, DC: Institute of Medicine, National Academy Press.

Lanier, D. and Clancy, C. 1997: Primary care research: current challenges, future needs. Journal of Family Practice 44, 434-38.

Nutting, P.A., Baier, M., Werner, J.J., Cutter, G., Reed, F.M. and Orzano, J.A. 1999: Practice patterns of family physicians in practice-based research networks: A report from ASPN. Journal of the American Board of Family Practice 12, 278-84.

Nutting, P.A., Beasley, J.W. and Werner, J.J. 1996: Practicebased research networks answer primary care questions. Journal of the American Medical Association 281, 686-88. 